\title{
HEURÍSTICA PARA EL EQUILIBRADO DE LÍNEAS DE ENSAMBLES DEL TIPO TSS
}

\section{HEURISTIC FOR THE TSS ASSEMBLY LINE BALANCING PROBLEM}

\author{
Pedro Palominos Belmar ${ }^{1} \quad$ Verónica Godoy Villalobos $^{2}$ \\ Recibido 11 de marzo de 2008, aceptado 15 de noviembre de 2010 \\ Received: March 11, 2008 Accepted: November 15, 2010
}

\begin{abstract}
RESUMEN
El propósito de este trabajo es presentar una heurística para el problema de equilibrado de líneas para células conformadas en "U". Particularmente para el sistema de fabricación denominado Toyota Sewing System (TSS), que representa un nuevo concepto de organización en una línea de producción. Si bien este tipo de sistema ha sido abordado en la literatura bajo el título de sistemas de producción autoequilibrado, en donde el equilibrio se logra mediante la utilización de dos reglas básicas, creemos que estas reglas no son suficientes para lograr el autoequilibrio. Por lo cual incorporamos el concepto de zonas de enlace que facilita el equilibrado de la línea. De los resultados obtenidos mediante simulación, concluimos que la heurística es eficiente para este tipo de sistemas de producción.
\end{abstract}

Palabras clave: Heurística, línea autoequilibrada, manufactura celular, sistema Toyota de costura, equilibrado de línea en $\mathrm{U}$.

\begin{abstract}
The purpose of this paper is to present a heuristic for the assembly line balancing problem in U-shaped cells, particularly for the manufacturing system called Toyota Sewing System (TSS), which represents a new production line organization concept. Although this type of system has been approached in the literature under the name of self-balanced production system, where the balance is achieved using two basic rules, we believe that these rules are not sufficient to achieve selfbalance, so we have incorporated the concept of linkage zones that facilitate balancing the line. From the results obtained by simulation we conclude that the heuristic is efficient for this kind of production system.
\end{abstract}

Keywords: Heuristics, self-balancing, celular manufacturing, Toyota sewing system, U-line Balancing.

\section{INTRODUCCIÓN}

La adopción de sistemas de producción tipo just-in-time (JIT) en la manufactura y en particular en la industria de la confección ha modificado las convencionales líneas de producción a líneas conformadas en "U", las cuales no pueden ser equilibradas mediante el tradicional enfoque de agrupar las tareas dentro de estaciones de trabajo a través de un grafo de precedencia. Esto se debe a que los trabajadores pueden operar en cualquier lado de la línea, ya que normalmente tiene un layout tipo "U", es decir, un trabajador puede hacerse cargo de las tareas iniciales o finales del proceso productivo no respetando las restricciones de precedencia de las tareas.
El problema simple de equilibrado de líneas en "U" (Uline assembly line balancing problem (UALBP)) fue modelado por primera vez por Miltenburg y Wijngaard (1994) [1], definiéndolo como una minimización del número de estaciones de trabajo $(\mathrm{N})$ para un tiempo de ciclo dado $(\mathrm{C})$, bajo las siguientes consideraciones:

Dados un conjunto de tareas $\mathrm{F}=\{\mathrm{k} / \mathrm{k}=1,2, \ldots ., \mathrm{n}\}$, un conjunto de restricciones precedentes $\mathrm{P}=\{(\mathrm{x}, \mathrm{y}) /$ la tarea $\mathrm{x}$ puede ser completada antes de la tarea $\mathrm{y}\}$, un conjunto de tiempos de ejecución de la tarea $\mathrm{T}=\{\mathrm{t}(\mathrm{k}) / \mathrm{k}=1,2, \ldots, \mathrm{n}\}$, y un tiempo de ciclo $\mathrm{C}$, encontrar una colección de subconjuntos de F, $(\mathrm{S} 1, \mathrm{~S} 2, \ldots, \mathrm{Sn})$, donde $\mathrm{Si}=\{\mathrm{k} /$ tarea $\mathrm{k}$ es hecha en la estación i . Es decir:

\footnotetext{
1 Departamento de Ingeniería Industrial. Universidad de Santiago de Chile. Casilla 10233. Santiago, Chile. E-mail: pedro.palominos@usach.cl

2 Escuela de Ingeniería Industrial. Universidad Adolfo Ibáñez. Balmaceda 1625. Viña del Mar, Chile. E-mail: vgodoy@uai.cl
} 
Minimizar $\quad\left[\mathrm{NC}-\sum_{\mathrm{i}=1}^{\mathrm{N}} \sum_{\mathrm{k} \in \mathrm{Si}} \mathrm{t}(\mathrm{k})\right]$

satisfaciendo las siguientes condiciones:

$$
\begin{aligned}
& \text { N } \\
& \cup \mathrm{Si}=\mathrm{F} \text {, } \\
& \mathrm{i}=1 \\
& \operatorname{Si} \cap \operatorname{Sj}=\varnothing, \\
& \sum \mathrm{t}(\mathrm{k}) \leq \mathrm{C}, \mathrm{i}=1,2, \ldots \ldots, \mathrm{N} \text {. }
\end{aligned}
$$

Para cada tarea y:

$\mathrm{Si}(\mathrm{x}, \mathrm{y}) \in \mathrm{P}, \mathrm{x} \in \mathrm{Si}, \mathrm{y} \in \mathrm{Sj}$, entonces $\mathrm{i} \leq \mathrm{j}$, para todo $\mathrm{x}$; o $\mathrm{Si}(\mathrm{y}, \mathrm{z}) \in \mathrm{P}, \mathrm{y} \in \mathrm{Sj}, \mathrm{z} \in \mathrm{Sk}$, entonces $\mathrm{k} \leq \mathrm{j}$, para todo $\mathrm{z}$.

La condición (2) asegura que todas las tareas son asignadas a alguna estación. Como resultado de la condición (3), cada tarea es asignada sola a una. Esto es, que el problema de equilibrado simple de una línea en U no permite tareas compartidas entre estaciones. La condición (4) asegura que el tiempo contenido de trabajo en una estación no exceda el tiempo de ciclo C. La condición (5) asegura que la restricción precedente no es violada en la línea en "U”. Como resultado de la condición (1), el número de estaciones de trabajo debe ser minimizado. Sin embargo, este nuevo planteamiento que modifica los modelos convencionales, en estructuras de subconjuntos ideales, no se ajusta necesariamente a la operatoria de funcionamiento del sistema tipo TSS (Toyota Sewing System), pues este nuevo sistema de trabajo viola la condición (3) planteada por Miltenburg y en consecuencia se debe buscar otra forma de abordar el problema de equilibrado, que es el objetivo del presente trabajo.

\section{EL MÉTODO TOYOTA SEWING SYSTEM (TSS)}

Este sistema fue desarrollado e instalado por primera vez en 1978 por la empresa Aisin Seiki Co. (miembro del grupo Toyota), y es conocido también con el nombre de zona de relevo [2] o línea con asistencia mutua [3] y en la literatura más técnica aparece con el nombre de sistemas de fabricación autoequilibrados [4-5], moving worker modules (MWM) [6]. Básicamente el TSS es de un grupo de personas que trabajan en equipo, con la particularidad de que sus áreas de trabajo, aunque en principio están definidas, sobre la marcha se van adaptando y reajustando a la rapidez y habilidad que demuestran los integrantes del grupo.

En la Figura 1 se presenta un layout típico del sistema Toyota, mostrándose los recorridos de los operadores $(\mathrm{A}, \mathrm{B}, \mathrm{C}, \ldots)$ por las distintas operaciones $(1,2,3, \ldots)$, que deben ser realizadas para fabricar una prenda. Se puede apreciar además que los recorridos se traslapan debido a la asistencia mutua entre trabajadores para equilibrar la línea de producción.

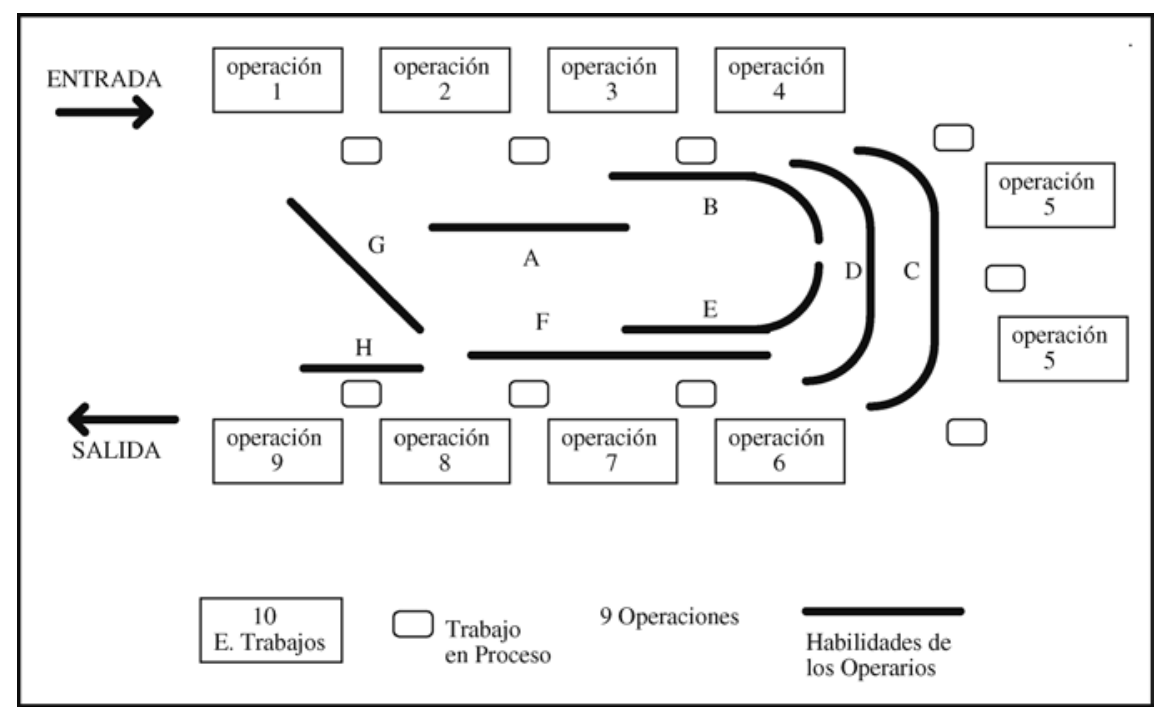

Figura 1. Layout típico del sistema Toyota [7]. 
Las reglas básicas del sistema de trabajo TSS [8] son las siguientes:

A. Un operador desempeña una operación en una estación y se mueve a la siguiente estación con la prenda en el sentido antihorario para ejecutar la próxima operación. El operador puede seguir moviéndose a la próxima estación desempeñando la operación respectiva, hasta encontrar otro operador en la estación. La prenda es entonces dejada frente a la estación o pasada directamente al operador si éste se encuentra libre.

B. Si un operador no está ocupado, el operador se moverá en sentido del reloj hasta encontrar una prenda disponible. Si no hay prendas en espera, el operador interrumpirá al primer operador y lo alcanza. El operador interrumpido se mueve en el sentido de reloj hasta encontrar una prenda disponible o interrumpir a otro operador que esté ocupado. El operador que interrumpe terminará la operación interrumpida.

En base a lo anterior Bartholdi (1996) [4] introdujo el concepto de líneas de producción autoequilibradas, demostrando matemáticamente que la tasa máxima de producción se alcanza si los trabajadores se ordenan en la secuencia asociada a la velocidad de los trabajadores, de menor a mayor velocidad en la ejecución de los trabajos. A esta nueva forma se denominó bucket brigades, que sería una instancia optimizada del sistema TSS [9]. No obstante, en la práctica no siempre es claro y evidente el poder discriminar la velocidad de los operarios en la realización de las operaciones, sobre todo cuando éstas son diversas y, en algunos casos, se producen cambios de modelos en los productos a fabricar en la línea.

Es evidente que en base a las dos reglas generales planteadas por Schroer (1991) [ 8] no se originaría el autoequilibrado de la línea en forma automática, ya que en la práctica se emplean reglas adicionales para implementar estos sistemas, utilizando el concepto de zonas de intercambio o enlaces, como una posta atlética, dado que tener a todos los operarios con el potencial de manejar todas las máquinas, así como ser capaces de realizar todas las operaciones que necesita el producto, es poco probable que ocurra en la práctica, ya que la polivalencia total es poco factible. Por lo tanto, existen reglas adicionales obtenidas a través de visitas a terreno [10], que ayudan a que el sistema TSS funcione adecuadamente. Las reglas son las siguientes:

i) El operario seguirá trabajando en la zona de relevo, hasta cuando venga el operario de los puestos posteriores; ii) El operario interrumpirá la operación que está realizando cuando venga el otro operario, quien seguirá con la operación. Por tanto, el operario que interrumpe la operación volverá a su primer puesto y sustituirá al operario de los puestos anteriores, en el caso de que esté trabajando en la zona de relevo;

iii) El operario no deberá trabajar o pasar más allá de la zona de enlace. En caso de que no venga el operario de los puestos posteriores a la zona de enlace, el operario debe volver a la cabeza del puesto que atiende y sustituir al otro en el caso que esté;

iv) Al volver a la cabeza del puesto que atiende el operario, al no estar otro y al no tener piezas preparadas para trabajar, espera hasta cuando venga el otro;

v) Al presentarse la espera por piezas preparadas, el encargado (líder de la línea) tomará las siguientes medidas:

a) Al presentarse siempre la espera, cambiar la zona de enlace.

b) Al presentarse de vez en cuando la espera, detectar posibles causas de fluctuaciones del tiempo de operación y tomar las medidas correctivas de cambios de zonas de enlaces.

vi) El volumen de trabajo en la zona de enlace será de 20-30\% del intervalo del tiempo de ciclo.

vii) La zona de enlace no significa siempre un puesto, pueden ser una parte de la operación de un puesto.

Como se puede observar, el problema de equilibrado de línea en este tipo de sistemas puede ser redefinido como un problema de definición de las zonas de enlace o intercambio, el cual será el enfoque del presente artículo, dado que los autores precedentes no abordan el tema desde esta perspectiva.

\section{UN NUEVO ENFOQUE PARA EL EQUILIBRADO DEL SISTEMA TSS}

Como se indicó en la introducción, la propuesta de Miltenburg [1] no es válida para equilibrar el sistema TSS, pues no se cumple la ecuación (2), $(\mathrm{Si} \cap \mathrm{Sj}=\varnothing$, con $i \neq j)$ al ser esta una línea de asistencia mutua y por lo tanto la ecuación (3) se transforma en:

$$
\begin{aligned}
& \mathrm{Si} \cap \mathrm{Sj} \neq \varnothing, \\
& i \neq j
\end{aligned}
$$

Esta intersección al no ser vacía significa que el operario $i$ excede en su recorrido al operario $j$ o que el operario $j$ coge parte del recorrido del operario $y$. Por tanto, se 
plantea una nueva forma de abordar este problema, mediante el estudio de las zonas de relevos en conjunto con los tiempos de ciclos.

Una primera aproximación al problema es la asignación de operaciones a los puestos de trabajo en forma secuencial, acercándonos lo más posible al tiempo de ciclo establecido (C), aunque será necesario definir las zonas de relevos o enlace para que el sistema funcione en su forma de autoequilibrio.

\section{Demarcación de zonas de enlace y redefinición del problema de equilibrado}

Como se ha indicado, en este sistema existe una autorregulación del equipo de operarios, definido por sus recorridos que se solapan con el fin de ajustarse a las tasas de producción definidas previamente. Estos solapamientos que se representan en la Figura 2 son zonas de enlace o intercambio, donde se entrega la prenda de un operario a otro. En esta figura se puede apreciar, por ejemplo, que el operario A deber hacer la operación 3 a la prenda; en caso de no poder, por alguna variabilidad propia de la operación o cadencia del operario, esta operación la ejecutará el operario B. De la misma forma se interpretan las otras zonas de la Figura 2.

\begin{tabular}{|c|c|c|c|c|c|c|c|}
\hline Proceso & & & & & & & \\
\hline $\mathrm{A}$ & $\mathrm{X}$ & $\mathrm{X}$ & $\mathrm{X}$ & & & & \\
\hline $\mathrm{B}$ & & & $\mathrm{X}$ & $\mathrm{X}$ & $\mathrm{X}$ & & \\
\hline $\mathrm{C}$ & & & & & $\mathrm{X}$ & $\mathrm{X}$ & $\mathrm{X}$ \\
\hline $\mathrm{D}$ & & & & & & & $\mathrm{X}$ \\
\hline
\end{tabular}

Figura 2. Zonas de intercambios de las prendas.

En consecuencia, el problema de equilibrado se puede reformular de la siguiente manera: “ ¿Cómo se asignan las zonas de enlace (donde se intercambian los productos), de tal forma que la línea de producción quede equilibrada?".

\section{Propuesta de una heurística al problema de equilibrado de línea para el sistema TSS}

La heurística propuesta tiene dos fases, la primera de ellas asigna las operaciones, o tareas, a las estaciones de trabajo, en forma consecutiva, hasta acercarse al tiempo de ciclo (C), y se basa en la heurística de Helgenson y Birnie (1961) [11]); luego, cuando la tarea j a ser asignada exceda el tiempo disponible de la estación, ésta se asignará a la estación actual o siguiente, en función del cálculo de $\Psi$, que representa la fracción del tiempo que la tarea j se realizaría en la actual estación, si esta tarea pudiese ser dividida para ser realizada en la estación actual y en la precedente.

La segunda fase de la heurística define las zonas de enlace en función del valor de $\Psi$. Aquí se definen la finalización de las tareas principales que son las que el operario debe desarrollar siempre; tareas parciales, las cuales el operario realiza a veces para ajustar el tiempo de ciclo, y finalmente las tareas eventuales que se ejecutan cuando ocurre algún evento inesperado que retrase el enlace con el próximo operario de la estación siguiente. Por tanto, las áreas de enlace quedan definidas por la intersección del conjunto de las estaciones.

Finalmente cabe señalar que el número de estaciones de trabajo para una célula y por consiguiente de operarios se consigue cuando el cuociente entre el tiempo total de las operaciones $\left(\Sigma \mathrm{t}_{\mathrm{i}}\right)$ y el tiempo de ciclo (C) es un número entero.

\section{$\Rightarrow$ Inicio de la heurística}

Dados un conjunto de tareas $\mathrm{F}=\{\mathrm{k} / \mathrm{k}=1,2, \ldots ., \mathrm{n}\}$, un conjunto de restricciones precedentes $\mathrm{P}=\{(\mathrm{x}, \mathrm{y}) /$ la tarea $\mathrm{x}$ puede ser completada antes de la tarea $y\}$, un conjunto de tiempos de ejecución de la tarea $\mathrm{T}=\{\mathrm{t}(\mathrm{k}) / \mathrm{k}=1,2, \ldots, \mathrm{n}\}, \mathrm{y}$ un tiempo de ciclo $\mathrm{C}$ para fabricar una prenda, se procede de la siguiente manera:

Primera Fase: Asignación de las operaciones a las estaciones de trabajo

Paso 1: Se abre la estación 1 y se le asigna el tiempo de ciclo que será el disponible.

Paso 2: Sea j la estación abierta y Td el tiempo disponible. Se asigna la tarea $k$ a la estación j, si se cumple con las siguientes condiciones:

Condición 1: no haber sido asignada todavía, Condición 2: tener las tareas precedentes inmediatas asignadas a una estación,

Condición 3: tener una duración inferior al tiempo disponible.

Si no hay más tareas que asignar de la lista de operaciones, vaya al paso 6.

Paso 3: Si no hay tareas que cumplan con las condiciones señaladas vaya al paso 5 .

Paso 4: Reducir el tiempo disponible en ti. Si el tiempo disponible es nulo defina $\Psi j=0$ y luego ir al paso 6 , en caso contrario ir al paso 2. 
Paso 5: Calcule la relación:

$$
\Psi=(T d) /(t k+1-T d)
$$

Siendo Td: tiempo disponible y tk+1: tiempo de la tarea $K+1$.

Si $\Psi>1$ asigne la tarea $k+1$ a la estación $j$. Defina $\Psi j=\Psi$ y luego vaya al paso 7.

Si $\Psi=1$ elija al azar la asignación de la tarea $k+1$ a la estación $j$. Si elige asignar la tarea $k+1$ a la estación $j$, y defina $\Psi j=1,5$ y luego vaya al paso 7. En caso contrario defina $\Psi j=0,5$ y vaya al paso 6 .

\{La asignación de $\Psi j=1,5$ y $\Psi j=0,5$ son indicativos, para definir las áreas de enlace en la fase 2.\}

Si $\Psi<1$ defina $\Psi j=\Psi$ y luego vaya al paso 6 .

Paso 6: Cerrar la estación j. Si todas las tareas están asignadas, el algoritmo ha terminado. En caso contrario abrir la estación $j+1$, asignándole el tiempo de ciclo como tiempo disponible e ir al paso 2. Si proviene del paso 5 asigne la tarea $k+1$ a la estación $j+1$, siendo el tiempo de esta tarea igual a :

$$
t^{\prime} k+1=t k+1-T d j
$$

vuelva al paso 4.

Paso 7: Cerrar la estación j. Si todas las tareas están asignadas el algoritmo ha terminado. En caso contrario abrir la estación $j+1, y$ asignarle como tiempo disponible para la estación $j+1$ el siguiente cálculo:

$$
T d j+1=C-(t k+1-T d j)
$$

vuelva al paso 2.

Una vez terminadas de asignar las tareas a las estaciones, se efectúa la segunda fase:

Segunda Fase: Definición de las áreas de enlace.

Paso 1: Se abre la estación j, y dependiendo del valor de $\Psi$ de la estación j asigne tareas como sigue:

Si $\Psi>1$ asigne a la estación j la primera tarea de la estación $j+1$ (que denominaremos tarea eventual), y asigne también la última tarea de la estación j a la estación $j+1$ (que denominaremos tarea parcial), luego vaya al paso 2.

Si $\Psi<1$ asigne a la estación j la primera tarea de la estación $j+1$ (que denominaremos tarea parcial), y asigne también la última tarea de la estación j a la estación $j+1$ (que denominaremos tarea eventual); luego vaya al paso 2.

Si $\Psi=0$ asigne a la estación j la primera tarea de la estación j+1 (que denominaremos tarea eventual), y asigne también la última tarea de la estación j a la estación j+1 (que denominaremos tarea parcial), luego vaya al paso 2.

Paso 2: Cerrar la estación j. Si la estación j+1 es la última cerrarla también e ir al paso 3. En caso contrario volver al paso 1.

Paso 3: Finalmente las zonas de enlace en la célula quedarán definidas como:

$$
\{E j\} \cap\{E j+1\}
$$

Siendo \{Ej\} el conjunto de tareas principales (fijadas en la fase 1), parciales y eventuales (fijadas en la fase 2), asignadas a la estación j.

\section{$\Rightarrow$ Fin de la heurística}

\section{MODELO DE SIMULACIÓN}

Para probar la eficiencia de la heurística propuesta se procedió a simular una célula de fabricación TSS descrita por Schroer, Wang and Ziemke [8], para la fabricación de pantalones femeninos de tejido de punto. La simulación fue programada en lenguaje Turbo Pascal 7.0. Los supuestos para la simulación fueron los siguientes:

- A cada operario se le asigna un conjunto de tareas respetando la precedencia de las mismas (recorrido).

- Pueden existir zonas de trabajo compartidas entre operarios adyacentes.

- Los operarios no se pueden adelantar.

- Los tiempos de operación se distribuyen normal, con una media y desviación estándar conocida.

- Los operarios tienen la misma eficiencia en cada operación.

- Cuando un operario traspasa un artículo al operario siguiente, se traspasa también el tiempo remanente de la tarea. 
- El tiempo de movimiento entre las distintas máquinas se asume cero.

- Las máquinas no tienen tiempo de inactividad.

- $\quad$ El abastecimiento de materiales es continuo.

\section{Parámetros y variables de salidas}

Para la operación del sistema se requieren los siguientes parámetros de entrada:

- Número de periodos de simulación.

- Duración de cada periodo.

- Número de corridas.

- Número de operaciones y duración (media y desviación estándar).

- Número de operarios y su recorrido.

Las variables de salida relevantes del modelo son las siguientes:

- Tasa de ocupación de cada operario en cada operación (máquina).

- Producción en cada periodo (productos terminados).

- Inventario promedio por periodo y por operación.

- Inventario en proceso al final de cada periodo por operación.

Como inventario por operación se considera el producto que se acumula a la salida de la operación y que no ha podido pasar a la operación siguiente.

\section{Aplicación}

En la Tabla 1 se presenta el número de identificación y el tiempo de ejecución de cada tarea para la fabricación de pantalones femeninos de tejido de punto [8].

Para este producto se aplicó la heurística propuesta, con tiempos de ciclo de 170, 135 y 100 segundos respectivamente, considerándose los siguientes escenarios:

A) Que los tiempos de las tareas sean determinísticos, ya que se desea saber cuál es la máxima tasa de producción sin variabilidad en los tiempos de fabricación.

B) Que los tiempos de las tareas se distribuyan normalmente con una desviación estándar equivalente al $15 \%$ del tiempo medio de operación.

C) Finalmente se consideró ampliar las zonas de intersección en los recorridos de los operarios, tomando en cuenta los recorridos resultantes de la simulación presentada en Schroer (1991) [8].
Tabla 1. Tareas para la fabricación de pantalón.

\begin{tabular}{|c|l|c|}
\hline Número & \multicolumn{1}{|c|}{ Tarea } & $\begin{array}{c}\text { Tiempo } \\
\text { (segundos) }\end{array}$ \\
\hline 01 & Coser bolsillos & 30 \\
\hline 02 & Pegar bolsillos a perneras & 70 \\
\hline 03 & Unir perneras & 60 \\
\hline 04 & Coser perneras & 40 \\
\hline 05 & $\begin{array}{l}\text { Coser abertura de } \\
\text { perneras }\end{array}$ & 95 \\
\hline 06 & Voltear prenda & 5 \\
\hline 07 & Hacer basta a la pierna & 40 \\
\hline 08 & Pegar banda elástica & 30 \\
\hline 09 & Acodar elástico & 5 \\
\hline 10 & Hacer ojal & 10 \\
\hline 11 & Puntada superior elástico & 15 \\
\hline 12 & Inspección & 20 \\
\hline 13 & $\begin{array}{l}\text { Empaquetado de la } \\
\text { prenda }\end{array}$ & 80 \\
\hline & & \\
\hline
\end{tabular}

Para los escenarios propuestos se simularon cuarenta periodos de cuatro horas que representan la operación de la célula durante cuatro semanas de cinco días, con jornadas de ocho horas diarias, divididas en segmentos de cuatro horas, con intermedio, situación que es representativa de las jornadas de trabajo en el sector de la confección.

Para el caso de un tiempo de ciclo $\mathrm{C}=170$ segundos, se puede calcular el número teórico de estaciones de trabajo. Este número se obtiene como el cuociente entre el tiempo total de las operaciones $\left(\sum \mathrm{t}_{\mathrm{i}}\right)$ y el tiempo de ciclo $(\mathrm{C})$. Si se obtiene un número entero, se satisface el tiempo de ciclo definido, en caso contrario, se debe aproximar al número al entero superior. En este caso, si $\Sigma \mathrm{t}_{\mathrm{i}}=500$ segundos, el número de trabajadores es 2,94 , es decir, tres trabajadores, teniendo un ocio de un $2 \%$.

Al aplicar la heurística, dio como resultado la siguiente asignación de tareas para los tres operarios definidos, que se muestran en la Tabla 2. Se puede notar la superposición de las tareas a ser realizadas por los operarios. Estas superposiciones son las zonas de enlaces donde se entrega el producto entre operarios para las siguientes operaciones a ser realizadas. 
Tabla 2. Distribución de operaciones para $\mathrm{C}=170$ segundos.

\begin{tabular}{|c|c|c|c|c|}
\hline $\begin{array}{c}\text { Opera- } \\
\text { ción }\end{array}$ & $\begin{array}{c}\text { Tiempo } \\
\text { (seg) }\end{array}$ & $\begin{array}{c}\text { Operario } \\
\mathbf{A}\end{array}$ & $\begin{array}{c}\text { Operario } \\
\mathbf{B}\end{array}$ & $\begin{array}{c}\text { Operario } \\
\mathbf{C}\end{array}$ \\
\hline 1 & 30 & $\mathrm{xxx}$ & & \\
\hline 2 & 70 & $\mathrm{xxx}$ & & \\
\hline 3 & 60 & $\mathrm{xxx}$ & $\mathrm{x}$ & \\
\hline 4 & 40 & $\mathrm{xx}$ & $\mathrm{xxx}$ & \\
\hline 5 & 95 & & $\mathrm{xxx}$ & \\
\hline 6 & 5 & & $\mathrm{xxx}$ & \\
\hline 7 & 40 & & $\mathrm{xxx}$ & $\mathrm{xx}$ \\
\hline 8 & 30 & & $\mathrm{x}$ & $\mathrm{xxx}$ \\
\hline 9 & 5 & & & $\mathrm{xxx}$ \\
\hline 10 & 10 & & & $\mathrm{xxx}$ \\
\hline 11 & 15 & & & $\mathrm{xxx}$ \\
\hline 12 & 20 & & & $\mathrm{xxx}$ \\
\hline 13 & 80 & & & $\mathrm{xxx}$ \\
\hline
\end{tabular}

Donde:

xxx: operación a ser realizada en forma total por el operario; $\mathbf{x x}$ : operación a ser desarrollada parcialmente por el operario y x: operación a ser desarrollada eventualmente por el operario.

\section{RESULTADOS Y ANÁLISIS}

A continuación se presentan los resultados del equilibrado para el caso un ciclo de 170 segundos y los tres escenarios propuestos.

\section{Primer escenario para un $\mathbf{C}=\mathbf{1 7 0}$ segundos}

Los resultados obtenidos con los tiempos de operación determinísticos se presentan en la Tabla 3.

Tabla 3. Utilización de los operarios (\%).

\begin{tabular}{|c|c|c|c|}
\hline Operación & $\mathbf{A}$ & $\mathbf{B}$ & $\mathbf{C}$ \\
\hline 1 & 18,00 & & \\
\hline 2 & 42,00 & & \\
\hline 3 & 35,99 & 0,01 & \\
\hline 4 & 4,01 & 19,99 & \\
\hline 5 & & 57,00 & \\
\hline 6 & & 3,00 & \\
\hline 7 & & 19,99 & 4,01 \\
\hline 8 & & 0,01 & 17,99 \\
\hline 9 & & & 3,00 \\
\hline 10 & & & 6,00 \\
\hline 11 & & & 9,00 \\
\hline 12 & & & 12,00 \\
\hline 13 & & & 48,00 \\
\hline TOTAL & $100 \%$ & $100 \%$ & $100 \%$ \\
\hline
\end{tabular}

La tasa de producción promedio por periodo fue 86,38 unidades, lo que equivale a un tiempo de ciclo de 166,72 segundos. No se produce acumulación de inventario, y al término de cada periodo permanece en el sistema un total de tres artículos, que equivalen al número de operarios. Todos los operarios permanecen $100 \%$ ocupados, ya que se trata de líneas con asistencia mutua que aseguran una alta utilización de los operarios, por lo que se obtiene la tasa de producción máxima.

Si se analiza la variabilidad en los tiempos de utilización de los operarios en los diferentes periodos, salvo en las estaciones de relevo, la desviación estándar es inferior al $1 \%$ del valor medio de utilización (el coeficiente de variación de los datos es mínimo).

Aun cuando los tiempos de operación son determinísticos, se presenta esta pequeña variabilidad en las tasas de utilización de los operarios en las diferentes operaciones, puesto que el periodo considerado para las estadísticas (cuatro horas) no coincide ni es un múltiplo del tiempo de ciclo, por lo que cada periodo comienza con el operario realizando una operación diferente.

\section{Segundo escenario para un $\mathbf{C}=\mathbf{1 7 0}$ segundos}

Asumiendo que los tiempos de operación son aleatorios, se obtuvieron los resultados de la Tabla 4.

Tabla 4. Utilización de los operarios (\%).

\begin{tabular}{|c|c|c|c|}
\hline Operación & $\mathbf{A}$ & $\mathbf{B}$ & $\mathbf{C}$ \\
\hline 1 & 17,92 & & \\
\hline 2 & 41,65 & & \\
\hline 3 & 31,81 & 3,82 & \\
\hline 4 & 8,62 & 15,20 & \\
\hline 5 & & 56,82 & \\
\hline 6 & & 2,98 & \\
\hline 7 & & 15,90 & 8,01 \\
\hline 8 & & 5,27 & 12,68 \\
\hline 9 & & & 2,98 \\
\hline 10 & & & 5,99 \\
\hline 11 & & & 8,99 \\
\hline 12 & & & 12,01 \\
\hline 13 & & & 47,86 \\
\hline TOTAL & $100 \%$ & $100 \%$ & $98,51 \%$ \\
\hline
\end{tabular}

La tasa de producción promedio por periodo fue 85,98 (unidades), variando de 84 a 87 unidades por periodo. Se produce un inventario promedio de 0,03 unidades, que se presenta en las estaciones de transferencia de artículos (4 y 8). 
Al final de cada periodo, permanece en el sistema un promedio de tres artículos. Aparece un tiempo ocioso en el último operario, quien presenta una menor flexibilidad en su ruta.

La línea presenta una eficiencia del 99,54\% respecto a la situación sin variabilidad. Cabe destacar que se produce una mayor intersección de los operarios en las zonas de transferencia.

\section{Tercer escenario para un $\mathbf{C}=\mathbf{1 7 0}$ segundos}

Considerando rutas ampliadas, los tiempos de utilización por operario se presentan en la Tabla 5.

En este caso, la tasa de producción promedio por periodo fue 86,30 unidades, variando de 84 a 88 unidades por periodo. Se produce un inventario promedio de 0,85 unidades, que se presenta en las estaciones 1,2 y 4 .

Al final de cada periodo, permanece en el sistema un promedio de 3,75 artículos. Aparece un tiempo ocioso en el operario intermedio. La línea presenta una mayor producción que en el caso anterior, pero sigue siendo inferior a la situación sin variabilidad.

Tabla 5. Utilización de operarios (\%).

\begin{tabular}{|c|r|r|r|}
\hline Operación & $\mathbf{A}$ & $\mathbf{B}$ & $\mathbf{C}$ \\
\hline 1 & 17,94 & & \\
\hline 2 & 39,22 & 3,01 & \\
\hline 3 & 25,49 & 10,44 & \\
\hline 4 & 11,41 & 12,47 & 0,06 \\
\hline 5 & 5,87 & 45,34 & 5,51 \\
\hline 6 & 0,03 & 2,21 & 0,76 \\
\hline 7 & 0,05 & 14,94 & 9,01 \\
\hline 8 & & 7,15 & 10,81 \\
\hline 9 & & 0,82 & 2,18 \\
\hline 10 & & 1,33 & 4,69 \\
\hline 11 & & 1,28 & 7,70 \\
\hline 12 & & 0,78 & 11,23 \\
\hline 13 & & & 48,06 \\
\hline TOTAL & $100 \%$ & $99,74 \%$ & $100 \%$ \\
\hline
\end{tabular}

\section{Resumen de resultados para los tiempos de ciclo estudiados}

En las Tablas 6, 7 y 8 se muestra el resumen de los resultados obtenidos para los tres escenarios propuestos.
Tabla 6. Resumen tiempo de ciclo $=170$ segundos.

\begin{tabular}{|l|c|c|c|}
\hline & $\begin{array}{c}\text { Escenario } \\
\mathbf{1}\end{array}$ & $\begin{array}{c}\text { Escenario } \\
\mathbf{2}\end{array}$ & $\begin{array}{c}\text { Escenario 3 } \\
\text { Schroer [8] }\end{array}$ \\
\hline $\begin{array}{l}\text { Producción } \\
\text { (unidades) }\end{array}$ & 86,38 & 85,98 & 86,30 \\
\hline Tiempo ocioso (\%) & 0,00 & 1,49 & 0,26 \\
\hline $\begin{array}{l}\text { Inventario promedio } \\
\text { (unidades) }\end{array}$ & 0,00 & 0,03 & 0,85 \\
\hline $\begin{array}{l}\text { Productos en } \\
\text { proceso (unidades) }\end{array}$ & 3,00 & 3,00 & 3,75 \\
\hline
\end{tabular}

Tabla 7. Resumen tiempo de ciclo $=135$ segundos.

\begin{tabular}{|l|r|r|l|}
\hline & $\begin{array}{c}\text { Escenario } \\
\mathbf{1}\end{array}$ & $\begin{array}{c}\text { Escenario } \\
\mathbf{2}\end{array}$ & $\begin{array}{l}\text { Escenario 3 } \\
\text { Schroer [8] }\end{array}$ \\
\hline $\begin{array}{l}\text { Producción } \\
\text { (unidades) }\end{array}$ & 112,20 & 108,05 & 107,18 \\
\hline Tiempo ocioso (\%) & 10,40 & 24,41 & 5,04 \\
\hline $\begin{array}{l}\text { Inventario promedio } \\
\text { (unidades) }\end{array}$ & 0,00 & 5,09 & 393,46 \\
\hline $\begin{array}{l}\text { Productos en } \\
\text { proceso (unidades) }\end{array}$ & 4,00 & 8,95 & 406,93 \\
\hline
\end{tabular}

Tabla 8. Resumen tiempo de ciclo $=100$ segundos.

\begin{tabular}{|l|c|c|c|}
\hline & $\begin{array}{c}\text { Escenario } \\
\mathbf{1}\end{array}$ & $\begin{array}{c}\text { Escenario } \\
\mathbf{2}\end{array}$ & $\begin{array}{c}\text { Escenario 3 } \\
\text { Schroer [8] }\end{array}$ \\
\hline $\begin{array}{l}\text { Producción } \\
\text { (unidades) }\end{array}$ & 144,00 & 139,58 & 111,38 \\
\hline Tiempo ocioso (\%) & 0,00 & 9,92 & 0,00 \\
\hline $\begin{array}{l}\text { Inventario promedio } \\
\text { (unidades) }\end{array}$ & 3,03 & 71,95 & $3.714,67$ \\
\hline $\begin{array}{l}\text { Productos en } \\
\text { proceso (unidades) }\end{array}$ & 8,00 & 78,93 & $3.812,43$ \\
\hline
\end{tabular}

A partir de los resultados anteriores, se puede comentar lo siguiente:

a) La heurística propuesta arroja buenos resultados en términos de tasa de producción, en relación a los resultados obtenidos mediante las dos reglas enunciadas por Schroer [8] y en donde los operarios tienen un mayor recorrido con los productos.

b) La tasa de utilización de los operarios se reduce en una situación con aleatoriedad, respecto de una situación sin aleatoriedad, cosa que es de esperar y que se refleja en una menor tasa de producción.

c) A medida que aumenta el número de operarios en la línea aumenta el inventario promedio y el número 
de artículos en proceso. El inventario en proceso se presenta principalmente antes de la estación 5, que es la estación "cuello de botella" (la estación con mayor tiempo de operación).

\section{CONCLUSIONES Y CONSIDERACIONES}

El presente trabajo es una contribución metodológica para la resolución del problema de equilibrado de células en "U", bajo el sistema de trabajo TSS, puesto que se reformula el problema de equilibrado y se propone una nueva heurística de solución basada en el uso de zonas de entrega o de intercambio de productos en procesos, siendo estas zonas una característica de los sistemas TSS.

Los resultados de la simulación muestran buenas soluciones para el caso estudiado en la literatura, validando de este modo la eficiencia de la heurística.

A futuro se espera estudiar el desempeño de la heurística, cambiando algunas reglas de decisión que se producen en las zonas de enlace o intercambio, de tal forma de saber si las reglas de decisión afectan el recorrido de los operadores en la línea de producción.

\section{AGRADECIMIENTOS}

Se agradece al Departamento de Investigaciones Científicas y Tecnológicas (DICYT) de la Universidad de Santiago de Chile, por el apoyo financiero para el proyecto 06017-PB.

\section{REFERENCIAS}

[1] G. Miltenburg and J. Wijngaard. "The U-Line Line Balancing Problem”. Management Science. Vol. 40, Issue 10, pp. 1378-1388. 1994.
[2] K. Suzaki. "Competitividad en la Fabricación en la Década de los 90”. TGP. Primera Edición Española. Madrid, España. 1990.

[3] H. Hirano. "El JIT Revolución en las Fábricas". TGP. Primera Edición Española. Madrid, España. 1990.

[4] J. Bartholdi and D. Eisenstein. "A production line balances itself”. Operations Research. Vol. 44, Issue 1, pp. 21-34. 1996.

[5] E. Zavadlav, J. McClain and J. Thomas. "Selfbuffering, self-balancing, self-flushing production lines". Management Science. Vol. 42, Issue 8, pp. 1151-1164. 1996.

[6] J. Chen and R. Askun. "Throughput maximization in serial production lines with worksharing". International Journal of Production and Economics. Vol. 99, Issue 1-2, pp. 88-101. 2006.

[7] National Economic Development Office (NEDO). "Team Working: A guide to modular manufacturing in the garment industries". NEDO. London, England. 1991.

[8] B.J. Schroer, J. Wang and M.C. Ziemke. "A look at TSS through simulation". Bobbin, pp. 114-119. July, 1991.

[9] A. Bractu and A. Dolgui. "A survey of the selfbalancing production lines ("bucket brigades")". Journal of Intelligent Manufacturing. Vol. 16, Issue 2, pp. 139-158. April, 2005.

[10] P. Palominos. "Contribución metodológica a la resolución del problema de equilibrado de células conformadas en "U" en la sección de costura: Una aproximación heurística”. Kurt Kauf. Barcelona, España. 1995.

[11] W.B. Helgenson and D. Birnie. "Assembly line balancing using ranked positional weight technique". Journal of Industrial Engineering. Vol. 12, pp. 394398. 1961. 